\title{
Sur la flore planctonique de Saint-Vaast-la-Hougue en 1907;
}

\author{
PAR M. L. MANGIN.
}

Les données relatives à la flore planctonique de l'Océan et de la Manche au voisinage des côtes de France font presque entièrement défaut. Nous ne possédons sur ce sujet que quelques indications fournies par CLEvE ${ }^{1}$ à la suite des envois de plancton qui lui avaient été faits par M. MALARD, sous-directeur du Laboratoire du Muséum à Tatihou, et les observations récemment publiées, par M. BERgon ${ }^{2}$ sur la flore diatomique du bassin d'Arcachon.

J'ai pensé qu'il était utile de combler cette lacune, en présence des documents si nombreux recueillis dans le Nord de l'Europe par Cleve, Gran, Ostenfeld, etc. Sur mes indications, M. Anthony, directeur-adjoint du Laboratoire de Tatihou à Saint-Vaast-laHougue, a fait procéder à des intervalles réguliers à des pêches de surface à environ un mille au large de Tatihou dans la direction des îles Saint-Marcouf.

Le produit des pêches en partie conservé dans l'alcool, en partie traité par des fixateurs variés (Brazil, Zenker, acide chromique) a été régulièrement adressé au laboratoire de Cryptogamie du Muséum tous les 15 jours, depuis le 15 février 1907, sauf au mois de novembre dernier où la perte des filets pendant une mer très forte a suspendu les envois.

C'est le résultat des observations fournies par l'examen de ces documents que je me propose de résumer ici. On verra, par la variété des formes et des faciès qui se sont succédé pendant presque une année, l'intérêt qui s'attache à ces observations et l'utilité qu'il y aurait à en multiplier le nombre en divers points des côtes de France.

1. Cleve (P. T.), The Plankton of the Nord Sea, the English Channel and the Skagerak in 1898), Kongl. svenska Vetenskaps-Akademiens Handlingar, Bandet 32, n० 8. Stockholm, 1900.

2. P. BERGon, Études sur la flore diatomique du bassin d'Arcachon et des parages de l'Atlantique voisins de cette station, Bull. Soc. scient. d'Arcachon, 1903. 
Pêche du 15 février 1907.

9 heures du matin. Pression 768. Température air $4^{\circ}$. Température mer $\breve{\Xi}^{\circ}$. Temps froid, brumeux, sans pluie. Vent du S.-0., forte brise ; mer assez agitée. - La semaine a été froide, grands vents N.-O. et $\mathbf{N}$. avec pluie.

1 Actinoptychus undulatus Ralfs ${ }^{1}$. 2 Asterionella japonica Cleve.

2 - kariana Grun.

4 Baccillaria paradoxa Gmel.

5 Biddulphia mobiliensis (Bail.)Grun

4 Chætoceros Diadema Ehr.

2 - curvisetum Cleve.

3 - densum Cleve.

5 - teres Cleve.

3 Coscinodiscus excentricus $E h r$.

1 - Oculus-Iridis Ehr.
1 Ditylium Brightwellii West.

1 Eucampia Zodiacus Ehr.

2 Guinardia flaccida Castr.

1 Lauderia annulata Cleve.

4 Melosira Borreri Grev.

2 Pleurosigma æstuarii W. Sm.

2 Skeletonema costatum Grev.

2 Thalassiosira gravida Cleve.

1 - Nordenskioldii Cleve

2 Thalassiothrix nitzschioides Grun.

\section{Pêche du $1^{\text {er }} \operatorname{mars} 1907$.}

9 heures matin. Pression 755 . Température air $6^{\circ}$. Température mer $5^{\circ}$. Temps brumeux, beau, sans pluie. Vent E. faible; mer calme.

1 Asterionella japonica Cleve.

1 Bacillaria paradoxa Grun.

1 Biddulphia mobiliensis Bail.

1 Chætoceros densum Ehr.

3 - Diadema Ehr.

1 - didymum $E h r$.

3 - curvisetum Cleve.

5 - teres Cleve.

3 Coscinodiscus excentricus Ehr.

1 - Oculus-Iridis Ehr.
1 Ditylium Brightwellii West.

1 Eucampia Zodiacus Ehr.

3 Lauderia annulata Cleve.

1 Melosira Borreri Grev.

1 Pleurosigma æstuarii W. Sm.

1 Rhizosolenia Shrubsolei Cleve.

2 Skeletonema costatum Grev.

5 Thalassiosira gravida Cleve.

5 - Nordenskioldii Cleve.

1 Thalassiothirix nitzschioidesGrun.

\section{Pêche du 15 mars 1907.}

9 heures du matin. Pression 768. Température air $8^{\circ}$. Température mer $7^{\circ}$. Temps froid avec pluie. Vent S.-0., forte brise; mer agitée. La mer était grosse depuis le $\mathbf{1}^{\mathrm{er}}$ mars avec vent froid et pluie. Vent $\mathrm{N}$. et N.-O.

1. Dans ces listes les chiffres qui précèdent les noms des diverses espèces indiquent la fréquence ou la rareté des individus, d'après l'échelle suivante dont on verra l'utilité dans le tableau résumant l'ensemble des pêches faites à St-Vaast en 1907 :

1 très rare, 2 rare, 3 peu commun, 4 assez commun, 5 commun, 6 très commun. 
1 Actinoptychus undulatus Ralfs.

1 Asterionella japonica Cleve.

2 - Kariana Grun.

4 Biddulphia mobiliensis Bail.

1 Bacillaria paradoxa tirun.

2 Chætoceros curvisetum Cleve.

2 - densum Ehr.

2 - Diadema Ehr.

1 - didymum $\mathbf{E h r}$.

2 - teres Cleve.

5 Coscinodiscus excentricus $E h r$.

2 - Oculus-Iridis $E h r$.
3 Ditylium Brightwellii West.

1 Eucampia Zodiacus Ehr.

1 Guinardia flaccida Castr.

1 Melosira Borreri Grev.

5 Lauderia annulata Cleve.

1 Nitzschia seriata Cleve.

2 Rhizosolenia Shrubsolei Cleve.

2 Skeletonema costatum Grev.

5 Thalassiosira gravida Cleve.

5 - Nordenskioldii Cleve.

1 Thalassiothrix nitzschioides Grun.

\section{Pêche du 29 mars 1907.}

9 heures du matin. Pression 768. Température de l'air 11․ Température de la mer $9^{\circ}$. Très beau temps. Vent N.-E.; mer très calme.

1 Biddulphia mobiliensis Bail.

1 Cerataulina Bergonii H. Perag.

1 Chætoceros densum $\mathrm{E} h r$.

1 - Diadema Ehr.

2 - curvisetum Ehr.

1 Coscinodiscus Oculus-Iridis $E h r$.

3 - excentricus $\boldsymbol{E} h \boldsymbol{r}$.
1 Eucampia Zodiacus Ehr.

6 Lauderia annulata Cleve.

1 Melosira Borreri Grev.

1 Pleurosigma æstuarii W. Sm.

5 Rhizosolenia Shrubsolei Cleve.

1 Pyrocystis Noctiluca Murray.

Pêche du 3 avril 1907.

9 heures du matin. Pression 750 . Température air $10^{\circ}$. Température mer $9^{\circ}$. Temps froid. Vent E. forte brise; mer très agitée. - Le temps était pluvieux les deux semaines précédentes et le vendredi 12, tempête de vent E., mer très grosse.

1 Actinoptychus undulatus Ralfs.

3 Biddulphia mobiliensis Bail.

4 Cerataulina Bergonii H. Perag.

2 Chætoceros densum Ehr.

3 - Diadema Ehr.

2 - curvisetum Cleve.

2 - teres Cleve.

2 Coscinodiscus excentricus $E h r$.

2 Eucampia Zodiacus $E h r$.
1 Guinardia flaccida Castr.

2 Lauderia annulata Cleve.

2 Leptocylindrus danicus Cleve.

1 Licmophora Lyngbyei Grun.

1 Melosira Borreri Grev.

5 Rhizosolenia Shrubsolei Cleve.

2 Thalassiosira gravida Cleve.

4 Pyrocystis Noctiluca Murray.

\section{Pêche du 26 avril 1907.}

9 heures du matin. Pression. Température air $11^{\circ}$. Température mer $9^{\circ}$. Temps brumeux. Vent 0 . faible; mer très belle. - Les deux semaines précédentes : temps beau avec mer belle. 
Le sédiment recueilli, peu abondant, était très pauvre en Diatomées, très riche en Crustacés.

2 Coscinodiscus excentricus Ehr.

2 Rhizosolenia Shrubsolei Cleve.

4 Pyrocystis Noctiluca Murray.

\section{Pêche du 10 mai 1907.}

9 heures du matin. Pression 759 . Température air $16^{\circ}$. Température mer $13^{\circ}$. Temps très beau, orageux. Vent faible, brise d'Est; mer calme. - Les deux semaines précédentes, pluie avec vent $\mathrm{S}$. et 0 .; mer agitée.

2 Chætoceros densum Ehr.

2 Guinardia flaccida Castr.

1 Melosira Borreri Grev.

4 Rhizosolenia Shrubsolei Cleve.

6 Pyrocystis Noctiluca Murray.

\section{Pêche du 24 mai 1907.}

9 heures du matin. Pression 756 . Température air $13^{\circ}$. Température mer $13^{\circ}$. Temps orageux avec pluie, vent E. faible; mer belle. - Les deux semaines précédentes beau temps, puis pluie fine avec mer belle.

1 Baccillaria paradoxa Grun.

3 Chætoceros curvisetum Cleve.

2 Guiryardia flaccida Castr.

1 Nitzs shia Closterium Ehr.
6 Rhizosolenia Shrubsolei Cleve.

1 - semispina Hensen.

3 -. Stolterfothii H. Perag.

\section{Pêche du 10 juin 1907.}

9 heures du matin. Pression 76 . Température air $16^{\circ}$. Température mer $14^{\circ}$. Mauvais temps avec pluie et orages. Vent S. et S.-0.; mer agitée. - Les deux semaines précédentes, mauvais temps; beaucoup de méduses.

1 Chætoceros densum Ehr.

1 Coscinodiscus excentricus Ehr.

1 Eucampia Zodiacus Ehr.

2 Guinardia tlaccida Castr.

1 Melosira Borreri Grev.
1 Nitzschia seriata Cleve.

6 Rhizosolenia Shrubsolei Cleve.

1 - semispina Hensen.

4 - Stolterfothii H. Perag.

Pêche du 26 juin 1907.

9 heures du matin. Pression 764. Température air $16^{\circ}$. Température mer $15^{\circ}$. Temps très mauvais avec pluie. Vent S.-0. très fort; mer très agitée. - Beaucoup de méduses.

1 Biddulphia mobiliensis Bail.

1 Chætoceros curvisetum Cleve.

2 - densum Ehr.

1 Guinardia flaccida Castr.
6 Rhizosolenia Shrubsolei Cleve.

1 - semispina Hensen.

2 - Stolterfothii H. Perag.

1 Pyrocystis Noctiluca Murray. 
Pêche du 9 juillet 1907.

9 heures du matin. Pression 767. Température air $14^{\circ}$. Température mer $14^{\circ}$. Temps froid avec pluie. Vent S.-0. fort; mer agitée. - Les deux semaines précédentes, même temps.

Sédiment peu abondant.

3 Guinardia flaccida Castr.

2 Chætoceros curvisetum Cleve.

2 Melosira Borreri Grev.

4 Rhizosolenia Shrubsolei Cleve.

3 Pyrocystis Noctiluca Murray.

\section{Pêche du 23 juillet 1907.}

9 heures du matin. Pression 762 . Température air $17^{\circ}$. Température mer $16^{\circ}$. - Des deux semaines précédentes, la première : très beau temps, mer belle, jolie brise de N.-E.; la deuxieme : temps brumeux avec pluie fine, mer calme, petite brise du $\mathbf{N}$. Le 22 juillet, fort orage avec pluie.

1 Chætoceros gracile Schütt.

1 Guinardia flaccida Castr.

1 Nitzchia seriata Cleve.
6 Rhizosolenia Shrubsolei Cleve.

1 - semispina Hensen.

2 - Stolterfothii H. Perag.

\section{Pêche du 5 août 1907.}

9 heures du matin. Pression 762. Température air $18^{\circ}$. Température mer $18^{\circ}$. Temps beau. Vent S.-0.; mer belle. - Mìme temps les deux semaines précédentes.

Sédiment peu abondant et très pauvre en Diatomées.

3 Guinardia flaccida Castr.

3 Rhizosolenia Shrubsolei Cleve.

1 - semispina Hensen
2 Rhizolenia Stolterfothii H. Perag.

2 Pyrocystis Noctiluca Murray.

\section{Pêche du 22 août 1907.}

9 heures du matin. Pression 752. Température air $20^{\circ}$. Température mer $19^{\circ}$. Temps beau. Faible brise 0 . et $\mathrm{N}$.; mer belle. - Les deux semaines précédentes, mème temps.

3 Guinardia flaccida Castr.

1 Nitzschia Closterium Ehr.

6 Rhizosolenia Shrubsolei Cleve.

1 Rhizosolenia semispina Hensen.

2 - Stolterfothii H.Perag.

\section{Pêche du 6 septembre 1907.}

9 heures du matin. Pression 768. Température air $19^{\circ}$. Température mer $18^{\circ}$. Temps beau. Vents 0 . et $\mathbf{N}$.- 0 ., belle brise; mer belle. - Le 
כ septembre, temps pluvieux, brumeux. - Temps beau et mer calme les deux semaines précédentes.

Sédiment peu abondant.

2 Guinardia flaccida Castr.

4 Rhizosolenia Shrubsolei Cleve.

2 Rhizosolenia Stolterfothii H.Perag.

\section{Pêche du 3 octobre 1907.}

9 heures du matin. Pression 752. Température air $18^{\circ}$. Température mer $17^{\circ}$. Très beau temps orageux. Vent faible du S. au S.-0.; mer calme. - Les semaines précédentes, beau temps jusqu'au 28 septembre ; puis forte pluie, vent du S., mer agitée.

1 Asterionella japonica Cleve.

1 Bacillaria paradoxa Grun.

3 Bacteriastrum varians Lauder.

1 Cerataulina Bergonii H. Perag.

6 Chætoceros curvisetum Cleve.

3 - densum $\boldsymbol{E} h r$.

4 - didymum Ehr.

2 - var. anglica.

1 Ditylium Brightwellii West.
2 Eucampia Zodiacus Ehr.

1 Eucampia spec.

3 Lauderia annulata Cleve.

1 Leptocylindrus danicus Cleve.

1 Nitzschia Closterium Ehr.

1 Pleurosigma æstuarii W. Sm.

1 Rhizosolenia Shrubsolei Cleve.

1 - semispina Hensen.

1 Thalassiosira gravida Cleve.

\section{Pêche du 21 octobre 1907.}

9 heures du matin. Pression 763. Température air $14^{\circ}$. Température mer $13^{\circ}$. Beau temps. Vent du S., faible brise; mer calme. - Les deux semaines précédentes, très mauvais temps avec pluie, vent d'abord du S. et S.-O., puis de l'E. et de l'O., mer très grosse.

Pêche faite à $2 \mathrm{~m}$. de profondeur.

1 Bacteriastrum varians Lauder.

6 Chrtoceros curvisetum Cleve.

4 - densum Ehr.

4 - didymum $E h r$.

2 - var. anglica.

3 - sociale Lauder.

3 Ditylium Brigtwellii West.

2 Eucampia Zodiacus Ehr.
2 Lauderia annulata Cleve.

1 Leptocylindrus danicus Cleve.

2 Nitzschia seriata Cleve.

1 Rhizosolenia Shrubsolei Cleve.

1 - Stolterfothii H. Perag.

2 Thalassiosira gravida Cleve.

1 Ceratium Fusus Duj.

1 Peridinium divergens $E h r$.

Pêche du 12 décembre 1907.

9 heures du matin. Pression 750 . Température air $6^{\circ}$. Température mer $4^{\circ}$. Vent très fort $\mathrm{S} .-0$., avec pluie; mer très agitée. Très mauvaị temps les deux semaines précédentes, vent S. et S.-0. très fort, mer très agitée; grande pluie. 
2 Asterionella japonica Cleve.

4 Biddulphia mobiliensis Bail.

2 Cerataulina Bergonii H. Perag.

4 Chætoceros densum Ehr.

2 - curvisetum Cleve.

6 - sociale Luuder forma typica.

4 - $\quad-\quad$ forma major.

3 Coscinodiscus Oculis Iridis Ehr.

2 - excentricus $\boldsymbol{E} h \boldsymbol{r}$.
3 Ditylium Brightwellii West.

1 Melosira Borreri Grev.

1 Nitzschia Closterium Ehr.

1 - seriata Cleve.

2 Rhizosolenia Shrubsolei Cleve.

2 - Stolterfothii H. Perag.

1 - semispina Hensen.

1 Thalassiosira gravida Cleve.

1 Thalassiothrix nitzschioides Grun.

Avant de commenter ces résultats, je voudrais les grouper en un tableau récapitulatif.

Les différents Mémoires qui traitent du plancton depuis les beaux travaux de C.Eve, présentent ces tableaux; mais leur lecture est difficile et il faut un certain temps pour découvrir dans chaque colonne les espèces dominantes, celles qui donnent à chaque pêche son caractère spécial. Les espèces sont en effet désignées par les lettres $r r, r, c, c c$, très rares, rares, communes ou très communes, qui ne sont pas très différentes à l'œil et n'accusent pas les différences qu'elles représentent. J'ai pensé qu'il conviendrait, pour rendre ces tableaux plus lisibles, de représenter chacune des espèces rencontrées dans une pêche par des signes conventionnels de même forme dont la grandeur varierait suivant le degré de fréquence ou de rareté et j'ai résumé le résultat des pêches dont la nomenclature précède au moyen de traits ou plutôt de rectangles ayant tous même base mais une hauteur d'autant plus grande que l'espèce est plus répandue; j’ai adopté six séries de ces rectangles ou mieux six traits d'épaisseur variable conformément à l'échelle ci-dessous.

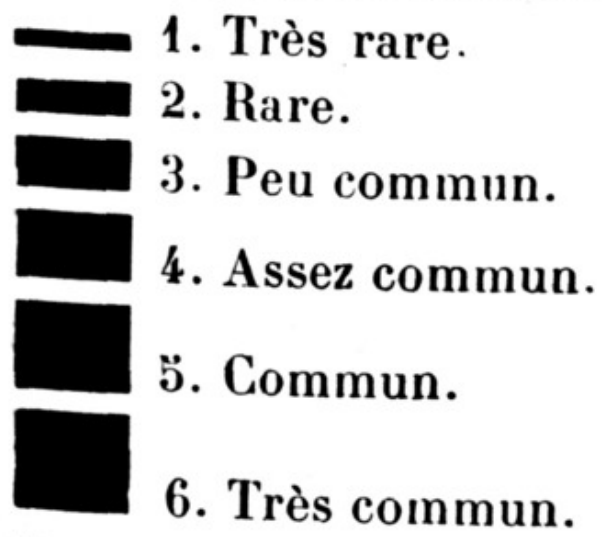

De celte manière, comme on peut s'en convaincre par l'examen du tableau résumant l'ensemble des pêches planctoniques de Saint-Vaast pendant l'année 1907, on distingue à première vue les espèces dominantes pour chaque pêche et le caractère homo- 
gène ou hétérogène de chacune d'elles se dessine avec netteté. Les tableaux deviennent ainsi très lisibles.

Pendant les mois de février et de mars, la flore planctonique est hétérogène et présente trois ou quatre espèces dominantes. Le 15 février, Coscinodiscus excentricus avec Chætoceros teres et Biddulphia mobiliensis dénote un mélange de formes néritiques tempérées et du Nord de l'Atlantique.

Le $1^{\text {er }}$ mars révèle une flore des côtes du Nord de l'Europe avec Thalassiosira Nordenskioldii et Th. gravida associés à Chrtoceros teres dont les individus étaient abondamment pourvus de spores durables.

Le 15 mars, Thalassiosira gravida et Thalassiosira Nordenskioldii diminuent un peu, Chætoceros teres est rare, mais Lauderia annulata, Biddulphia mobiliensis, très abondants, accusent la prédominance des formes néritiques tempérées.

Le 29 mars, la flore s'appauvit. Lauderia annulata est encore abondant, et nous voyons apparaître Rhizosolenia Shrubsolei qui, très abondant et accompagné de quelques espèces rares, donne une physionomie de flore néritique tempérée caractéristique.

Le 13 avril, la flore conserve son caractère tempéré, et Rhizosolenia Shrubsolei domine avec Cerataulina Bergonii, en même temps apparaît en abondance Pyrocystis Noctiluca qui va devenir prédominant le 10 mai et constituer une flore très homogène; à partir du 14 mai et jusqu'au 6 septembre, Rh́izosolenia Shrubsolei domine, désormais accompagné, en plus ou moins grande abondance, par Rhizosolenia Stolterfothii, plus rarement par Guinardia flaccida, Rhizosolenia semispina et parfois par Pyrocystis Noctiluca.

Au mois d'octobre, le plancton change d'allures et se distingue par l'abondance et la variété des Chxtoceros parmi lesquels Chæloceros curvisetum est dominant et constitue la caractéristique de ce plancton homogène. C'est un plancton du Nord de l'Europe associé à des espèces néritiques tempérées, Lauderia annulata et Bacteriastrum varians ${ }^{1}$.

Au mois de décembre, le plancton est toujours riche en $C h x$ toceros, mais le Ch. curvisetum a beaucoup diminué et c'est le

1. Un accident, la perte des filets du laboratoire pendant une tempête, m'a privé des péches de novembre qui eussent été intéressantes. 
1907. - FLORE PLANCTONIQUE DE SAINT-VAAST-LA-HOUGUE

\begin{tabular}{|c|c|c|c|c|c|c|c|c|c|c|c|c|c|c|c|c|c|c|}
\hline & $\frac{15}{2}$ & $\frac{1}{3}$ & $\frac{15}{3}$ & $\frac{29}{3}$ & $\frac{13}{4}$ & $\frac{26}{4}$ & $\frac{10}{5}$ & $\frac{24}{5}$ & $\frac{10}{6}$ & $\frac{26}{6}$ & $\frac{7}{7}$ & $\frac{23}{7}$ & $\frac{5}{8}$ & $\frac{22}{8}$ & $\frac{6}{9}$ & $\frac{3}{10}$ & $\frac{21}{10}$ & $\frac{12}{12}$ \\
\hline Actinoptychus undulatus (Bail?) Ralfs. & & & & & & & & & & & & & & & & & & \\
\hline Asterionella japonica Clere........... & & & & & & & & & & & & & & & & & & \\
\hline Asterionella kariana Grun.. & & & & & & & & & & & & & & & & & & \\
\hline Bacillaria paradoxa (fmel) Grun.. & & & & & & & & & & & & & & & & & & \\
\hline Bacteriastrum varians Lauder... & & & & & & & & & & & & & & & & & & \\
\hline Biddulphia mobiliensis Bail... & & & & & & & & & & & & & & & & & & \\
\hline Cerataulina Bergonii $H$. Per..... & & & & & & & & & & & & & & & & & & \\
\hline Chætoceros curvisetum Cleve.. & & & & & & & & & & & & & & & & & & \\
\hline Chætoceros densum $E h r . . .$. & & & & & & & & & & & & & & & & & & \\
\hline Chætoceros Diadema Ehr.... & & & & & & & & & & & & & & & & & & \\
\hline Chætoceros didymum Ehr.... & & & & & & & & & & & & & & & & & & \\
\hline Chætoceros didymum var anglica... & & & & & & & & & & & & & & & & & & \\
\hline Chætoceros gracile Schüt... & & & & & & & & & & & & & & & & & & \\
\hline Chætoceros teres Cleve...... & & & & & & & & & & & & & & & & & & \\
\hline Chætoceros sociale Lauder... & & & & & & & & & & & & & & & & & & \\
\hline Coscinodiscus excentricus $E h r \ldots \ldots$ & & & & & & & & & & & & & & & & & & \\
\hline Coscinodiscus Oculus-Iridis Ehr.... & & & & & & & & & & & & & & & & & & \\
\hline Ditylium Brightwellii (Wesl) Grun.. & & & & & & & & & & & & & & & & & & \\
\hline Eucampia Zodiacus $E h r \ldots \ldots \ldots \ldots$ & & & & & & & & & & & & & & & & & & \\
\hline Guinardia flaccida Cast.. . & & & & & & & & & & & & & & & & & & \\
\hline Lauderia annulata Cleve.... & & & & & & & & & & & & & & & & & & \\
\hline Leptocylindrus danicus Cleve...... & & & & & & & & & & & & & & & & & & \\
\hline Licmophora Lyngbyei (Kütz.) Gr'un. & & & & & & & & & & & & & & & & & & \\
\hline Melosira Borreri Grev............. & & & & & & & & & & & & & & & & & & \\
\hline Nitzschia Closterium E/r..... & & & & & & & & & & & & & & & & & & \\
\hline Nitzschia seriata Cleve.......... & & & & & & & & & & & & & & & & & & \\
\hline Pleurosigma æstuarii $\mathrm{W} . \mathrm{s} m .$. & & & & & & & & & & & & & & & & & & \\
\hline Rhizosolenia Shrubsolei Cleve... & & & & & & & & & & & & & & & & & & \\
\hline Rhizosolenia semispina Hensen...... & & & & & & & & & & & & & & & & & & \\
\hline Rhizosolenia Stolterfothii $H$. Perrg... & & & & & & & & & & & & & & & & & & \\
\hline Skeletonema costatum Grev. & & & & & & & & & & & & & & & & & & \\
\hline Thalassiosira gravida Cleve.... & & & & & & & & & & & & & & & & & & \\
\hline Thalassiusira Nordenskiöldii Cleve.. & & & & & & & & & & & & & & & & & & \\
\hline Thalassiuthrix nitzschioides Grun... & & & & & & & & & & & & & & & & & & \\
\hline Pyrocystis Noctiluca Murray......... & & & & & & & & & & & & & & & & & & \\
\hline Ceratium Fusus $D u j \ldots \ldots \ldots \ldots \ldots$ & & & & & & & & & & & & & & & & & & \\
\hline Peridinium divergens $E h r . .$. & & & & & & & & & & & & & & & & & & \\
\hline
\end{tabular}


Chætoceros sociale, dont l'apparition avait été signalée à la fin d'octobre, qui constitue l'espèce dominante. Les colonies de cette espèce sont très nombreuses et à tous les états de développement.

On voit, en somme, que la flore de Saint-Vaast s'est montrée particulièrement riche en Diatomées dont les types caractéristiques, au nombre de quatre, se sont succédé pendant des périodes d'inégale importance, Thalassiosira Nordenskioldii et Th. gravida en mars avec Chætoceros teres; Rhizosolenia Shrubsolei pendant tout l'été, puis, en octobre, Chætoceros curvisetum et, en décembre, Chætoceros sociale.

J'ai représenté dans la planche I les aspects caractéristiques de ces diverses flores par des photographies de préparations microscopiques.

Les Péridiniens sont pour ainsi dire absents de la flore de Saint-Vaast. A l'exception du Pyrocystis Noctiluca qui a présenté son maximum d'importance au mois de mai, les vrais Péridiniens manquaient dans les sédiments examinés; j’ai seulement rencontré çà et là, et par échantillons toujours très rares, Peridinium divergens et Ceratium Fusus.

Il serait prématuré de tirer des conclusions de la succession des flores que je viens de signaler. Nous devons nous borner maintenant à enregistrer les résultats des analyses de pêches périodiques recueillies pendant un certain nombre d'années; quand le nombre des pêches deviendra assez considérable, il sera possible, en les coordonnant, de déduire des données importantes sur la loi de répartition des espèces. Toutefois, les résultats fournis par CLEVE en 1899 me permettront d'établir une courte comparaison avec les miens. Parmi les espèces mentionnées pạr cet auteur à Saint-Vaast-la-Hougue, le Rhizosolenia s'était montré abondant pendant une période un peu plus courte qu'en 1907, du milieu de juin au milieu du mois d'août; nous l'avons constaté très abondant depuis le mois de mai jusqu'en septembre. 11 y a concordance pour la distribution du Chætoceros curvisetum; par contre, les Thalassiosira Nordenskioldii et gravida, espèces néritiques du Nord de l'Europe, ne sont pas signalées à Saint-Vaast, pas plus qu'à Plymouth, bien que l'abondance du Chætoceros teres ait été mentionnée en janvier 1899; le Rhizo- 


\section{$2 \mathrm{BHL}$ Biodiversity Heritage Library}

Mangin, M L . 1908. "Sur la flore planctonique de Saint-Vaast-la-Hougue en 1907." Bulletin de la Société botanique de France 55, 13-22.

https://doi.org/10.1080/00378941.1908.10831328.

View This Item Online: https://www.biodiversitylibrary.org/item/8677

DOI: https://doi.org/10.1080/00378941.1908.10831328

Permalink: https://www.biodiversitylibrary.org/partpdf/160680

\section{Holding Institution}

Missouri Botanical Garden, Peter H. Raven Library

\section{Sponsored by}

Missouri Botanical Garden

\section{Copyright \& Reuse}

Copyright Status: Public domain. The BHL considers that this work is no longer under copyright protection.

This document was created from content at the Biodiversity Heritage Library, the world's largest open access digital library for biodiversity literature and archives. Visit BHL at https://www.biodiversitylibrary.org. 